Check for updates

Cite this: Mater. Adv., 2021,

2. 1343

Received 27th November 2020

Accepted 14th January 2021

DOI: 10.1039/d0ma00930j

rsc.li/materials-advances

\section{The impact of lipid head-groups in GUVs on electron transfer by surface-adsorbed fluorescent gold nanoclusters $\dagger$}

\begin{abstract}
Arunavo Chatterjee and Pradipta Purkayastha (D) *
Glutathione-coated gold nanoclusters (GSH-AuNCs) have immense medical importance. Being photoinduced electron transfer (PET) facilitators, these nanomaterials are useful as photosenstizers and applied in photocatalysis, radiotherapy, tomography imaging, etc. GSH-AuNCs interact with the lipid bilayer of the cellular membrane and take part in drug delivery and functioning. Since PET is a key player here, hence, understanding the phenomenon in detail is extremely necessary. Herein, we have synthesized GSH-protected orange emitting AuNCs and analyzed their interactions with giant unilamellar vesicles (GUVs) made from lipids, namely, DMTAP and DPPC, with positive and zwitterionic head groups, respectively. The photophysical changes in the process were monitored by steady state and time resolved fluorescence spectroscopy to interpret the possible binding of GSH-AuNCs with lipid vesicles. The AuNCs are hydrophilic and hence adsorb over the surface of GUVs. A well-known electron scavenger, methyl viologen $\left(\mathrm{MV}^{2+}\right)$ was added externally to the system to trigger PET with the GSHAuNCs at neutral $\mathrm{pH}$. The dynamics were analyzed by the method of fluorescence quenching due to PET. It is found that attachment of GSH-AuNCs with lipid vesicles having differently charged head groups is very essential in deciding the extent of PET since DMTAP GUVs promote PET to a greater extent compared to DPPC GUVs. The experimental conclusions were supported by theoretical studies. The kinetic and thermodynamic parameters of the PET process were also calculated.
\end{abstract}

\section{Introduction}

Glutathione-coated gold nanoclusters (GSH-AuNCs) are important nanomaterials that have gained their significance because of the protecting or stabilizing agent. GSH ligands help AuNCs to dissolve in water due to the presence of carboxylate groups. Moreover, their terminal carboxylic acid groups help AuNCs to interact with many molecules for a vivid range of applications. $^{1-3}$ These ultrasmall GSH-AuNCs, ranging from 1 to $2 \mathrm{~nm}$ in diameter, do not cause any biological toxicity even up to $60 \mu \mathrm{M}$ concentration. ${ }^{4}$ Reports on small non-fluorescent GSH-coated $\mathrm{Au}$ nanoparticles (AuNPs) with 1.5-2.5 nm diameter demonstrate renal clearance and tumor accumulation properties. ${ }^{5,6}$ GSH-protected AuNCs and/or AuNPs bear negative surface charge under physiological conditions and it has been noted that compared to zwitterionic or PEGylated nanoparticles, they potentially bind to serum proteins in biological fluids that have high ionic strength. ${ }^{7-9}$ GSH-AuNCs are sensitive to the $\mathrm{pH}$ of the

Department of Chemical Sciences, Indian Institute of Science Education and Research (IISER) Kolkata, Mohanpur 741246, WB, India.

E-mail: ppurkayastha@iiserkol.ac.in

$\dagger$ Electronic supplementary information (ESI) available: Experimental details and additional figures. See DOI: 10.1039/d0ma00930j medium as well. Lowering the pH allows GSH to get converted to its zwitterionic form that initiates self-aggregation. ${ }^{10-12}$ All these factors are sensible for the functioning of the well-segregated GSH-AuNCs in the biological medium. Only under such conditions it becomes feasible to exploit their photocatalytic and photosensitizing properties in photodynamic therapy (PDT). For example, GSH-AuNCs loaded over $\mathrm{SiO}_{2}, \mathrm{TiO}_{2}$, or inorganic NPs enhance their photostability as well as photoredox potency. ${ }^{13-16}$ Evidences of efficient photosensitization by GSH-AuNCs have been reported in oxygen, hydrogen, and singlet oxygen generation. ${ }^{17,18}$ NPs or NCs can preserve their dispersion in biological membranes helping in efficient interactions. ${ }^{19}$ Reports are there on such interactions with lipid rafts, ${ }^{20}$ bilayers, ${ }^{21}$ and vesicles. $^{22}$ Herein, we have attempted a similar approach to trap GSH-AuNCs on lipid vesicles with different surface charge made from 1,2-dimyristoyl-3-trimethylammonium-propane (DMTAP) and dipalmitoylphosphatidylcholine (DPPC) (Scheme 1). Negative charges on GSH-AuNCs make them prone to adsorption on the surface of the lipid vesicles and hence the photosensitization of NCs can be probed by a suitable electron scavenger, such as methyl viologen $\left(\mathrm{MV}^{2+}\right)$.

An extensive review by Bain et al. has shown that metal NCs are used to make hybrid systems for energy harvesting and sensing. ${ }^{23}$ 


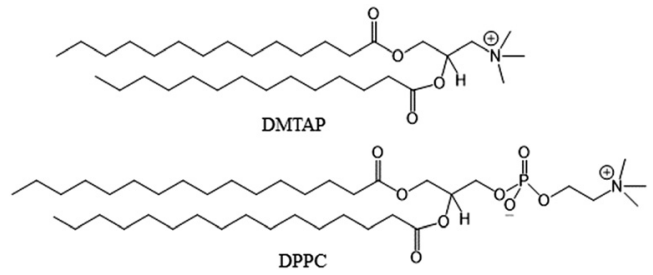

Scheme 1 Structures of DMTAP and DPPC with the charges of the head groups.

Photoinduced electron transfer (PET) is quite common in biocatalysis ${ }^{24}$ as well as in singlet oxygen generation. ${ }^{18}$ In this context, the GSH-AuNCs are quite useful in visible lightinduced hydrogen formation and light harvesting. ${ }^{17,25}$ Hence, we used PET as a tool to investigate the unventured area of the effect of differently charged head groups of lipids (in the form of vesicles) on the extent of PET between the surface adsorbed GSH-AuNCs and externally added $\mathrm{MV}^{2+}$ ions (electron acceptor). It is seen that charge distribution at the lipid heads substantially controls the extent of PET between the donor-acceptor pair. We have calculated the results using steady state and timeresolved spectroscopy. The findings were supported by theoretical (density functional) calculations.

\section{Experimental section}

\subsection{Synthesis of the GSH-protected AuNCs}

The tripeptide GSH was used as the stabilizing as well as reducing agent to prepare orange-emitting AuNCs following a slightly modified reported method. ${ }^{26}$ Briefly, an aqueous solution of $\mathrm{HAuCl}_{4}(1 \mathrm{~mL}, 20 \mathrm{mM})$ was mixed with reduced GSH $(0.3 \mathrm{~mL}, 100 \mathrm{mM})$ in distilled water. The resulting yellow solution was stirred for few minutes to obtain a turbid substance at room temperature, which eventually became clear. This was heated at $70{ }^{\circ} \mathrm{C}$ for $24 \mathrm{~h}$ under gentle stirring. The resultant greenish yellow solution was kept at $40{ }^{\circ} \mathrm{C}$ for further use. The solution emitted orange light on illumination with UV radiation. The concentration of the GSH-AuNCs was kept at $20 \mu \mathrm{M}$ throughout the experiment.

\subsection{Synthesis of the giant unilamellar vesicles (GUVs)}

Lipids having cationic (DMTAP) and zwitterionic (DPPC) head groups were chosen for this experiment. The GUVs were synthesized following a reported method. ${ }^{27}$ Briefly, weighed amounts of the lipids were dissolved separately in a $2: 1$ mixture of chloroform and methanol to make the total volume $800 \mu \mathrm{L}$. This solution was vortexed for a few minutes and sonicated in a bath sonicator for complete dissolution of the lipids. The resulting solutions were hydrated carefully along the walls of each of the round bottom flasks with $5 \mathrm{~mL}$ of tris buffer at $\mathrm{pH}$ 7.4. The total lipid concentration was fixed at $0.25 \mathrm{M}$ in each case. The hydrated solutions were rotated under reduced pressure to evaporate the organic part and opalescent solutions of $5 \mathrm{~mL}$ each were obtained. Confocal images show that the diameter of the lipid vesicles is about $220 \mu \mathrm{m}$, which confirms that these are GUVs (Fig. S1, ESI $\dagger$ ).

\section{Results and discussion}

\subsection{Characterization of the GSH-AuNCs}

The synthesized GSH-AuNCs were characterized by different techniques including microscopy and spectroscopy. The transmission electron microscopy (TEM) images show that the AuNCs are 1-1.5 nm in diameter (Fig. 1A). Their hydrodynamic diameter is $\sim 16 \mathrm{~nm}$ (Fig. $1 \mathrm{~B}$ ) and the average zeta potential is about $-14.8 \mathrm{mV}$ at $\mathrm{pH} 7.4$ (tris buffer) (Fig. 1C). The absorption spectrum of the GSH-AuNCs shows a broad shoulder at $\sim 400 \mathrm{~nm}$; their emission peaks at $600 \mathrm{~nm}$ and the excitation spectrum shows a prominent shoulder at $\sim 400 \mathrm{~nm}$ (Fig. 2).

Emission from the GSH-AuNCs is mainly due to (i) the core state transitions between the $\mathrm{Au}(\mathrm{I})$ and $\mathrm{Au}(0)$ centers and (ii) the ligand-to-metal charge transfer (LMCT) between the negativelycharged GSH molecules and the metallic core. A $200 \mathrm{~nm}$ Stokes shift (Fig. 2) signifies the overwhelming involvement of the LMCT states in the emission. ${ }^{28}$ The excited core states rapidly decay to the ground state or relax non-radiatively to the CT state, nurturing the LMCT transition. Decay of the LMCT state is quite slow. Time resolved fluorescence spectroscopy of the GSH-AuNCs, therefore, shows three components fitted to the raw data, two of which are quite fast owing to the transitions arising from the excited core states ( 0.5 and $7 \mathrm{~ns}$ ) and the other one is much slower (119 ns), depicting the transition from the longer-lived LMCT states. ${ }^{28}$ The fluorescence quantum yield of
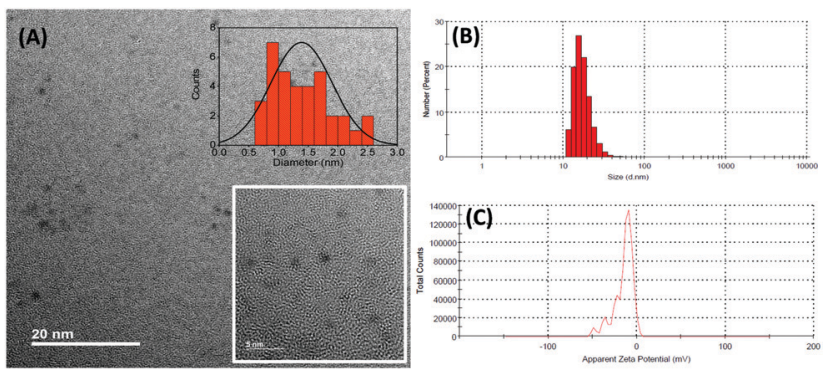

Fig. 1 (A) TEM images of the GSH-AuNCs. The insets show the size distribution of the AuNCs and the HR-TEM image; (B) the hydrodynamic diameter and (C) the average zeta potential of the GSH-AuNCs.

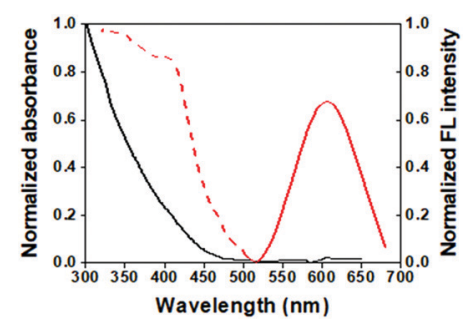

Fig. 2 Spectroscopic characterization of the GSH-AuNCs: the absorption (solid, black), emission (solid, red) $\left(\lambda_{\mathrm{ex}}=375 \mathrm{~nm}\right)$, and excitation (dash, red) spectra. 
the GSH-AuNCs is about 3\% as measured with respect to the quinine sulphate (QY 0.54) in $0.1 \mathrm{M} \mathrm{H}_{2} \mathrm{SO}_{4}$ standard.

\subsection{Interaction of the GSH-AuNCs with GUVs}

We initially looked into the interaction of the GSH-AuNCs with the lipid vesicles before moving into the application of the electron scavenger. This is important because the lipid vesicles that mimic the biological cells may induce special properties to the adsorbed AuNCs to influence any electron transfer phenomenon. It was explicitly shown by $\mathrm{Wu}$ et al. that the surface ligands contribute largely to enhance the fluorescence of AuNCs. ${ }^{29}$ Two ways of influencing the AuNC fluorescence are the occurrence of LMCT directly or through the $\mathrm{Au}-\mathrm{S}$ bonds. We have shown direct evidence to the report of $\mathrm{Wu}$ et al. through our experiment, which showed variations in the fluorescence of GSH-AuNCs with negative surface charge on interactions with DMTAP and DPPC GUVs bearing different charges on the lipid head groups. The emission spectrum of the GSH-AuNCs shows a steady rise in intensity on interacting with the DMTAP GUVs until saturation (Fig. 3A) with practically no change in the corresponding absorption spectrum (not shown). On the other hand, on interacting with the DPPC GUVs, the fluorescence intensity grossly decreases with an increase in the lipid concentration (Fig. 3B). The enhancement of fluorescence in case of the DMTAP GUVs could presumably be due to greater degree of LMCT arising because of the interaction of the GSH-AuNCs with DMTAP GUVs (Fig. 3C). The quaternary ammonium groups of the lipid molecules interact electrostatically with the negatively charged surface of the GSH-AuNCs promoting excited state LMCT. In case of the DPPC GUVs, the fluorescence intensity increased initially on adding $2.5 \mu \mathrm{M}$ lipid and subsequently decreased on further addition of the DPPC GUVs. Such a behavior of the GSH-AuNCs is expected since initially a certain population of the AuNCs (presumably similar for both the lipids) adsorb on the surface of the GUVs, showing an increase in the fluorescence intensity due to motional
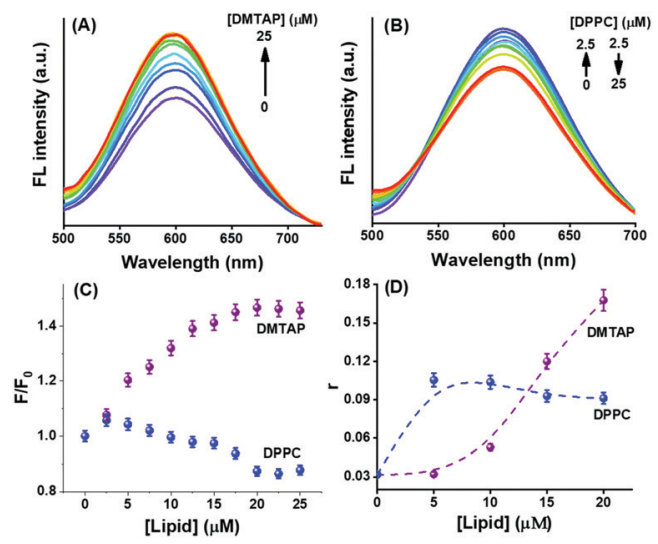

Fig. 3 Influence of the added GUVs from (A) DMTAP and (B) DPPC lipids on the fluorescence emission of the GSH-AuNCs in aqueous buffer under normal conditions ( $\lambda_{\mathrm{ex}}=375 \mathrm{~nm}$ ); (C) provides a comparative plot of the relative fluorescence yields due to the interactions and (D) shows the steady state fluorescence anisotropy ( $r$ ) plots for the two cases (the dashed lines are to guide the view). restriction. Since the interaction of the negative surface of the GSH-AuNCs and the positive surface of the DMTAP GUVs is supposedly stronger compared to that with the zwitterionic DPPC GUVs, hence, the adsorption of the AuNCs progressively increases until saturation with an increase in the GUV population. However, further addition of DPPC GUVs offers more adsorbing substrates to the GSH-AuNCs allowing some of the previously adsorbed AuNCs to get redistributed. Weaker binding allows them to spread over more substrates, resulting into a decrease in the fluorescence intensity. The reason behind the variations in the fluorescence emissions for DMTAP and DPPC GUVs was proved to be due to the alteration in charge distribution at the lipid head groups in the excited states that regulated the LMCT and hence the emission intensity. The difference in the nature of surface adsorption of the GSH-AuNCs on the DMTAP and DPPC GUVs influenced the change in the fluorescence emission of the AuNCs differently in the two cases. It becomes clear that the greater population of GSH-AuNCs on the GUV surface will influence LMCT more since the charge transfer, which was taking place from GSH to the AuNC core before interaction with the GUVs, will be strongly affected by the excited state charge accumulation at the lipid heads attached to the GSH-AuNCs. Since the population of the adsorbed GSH-AuNCs is lower in the case of DPPC and the charge accumulation at the lipid heads is receded (vide infra), hence, the GSH ligands will be more concentrated in the electrostatic binding process than in the charge transfer to the AuNC core.

The variation in the AuNC distribution over the GUVs with different surface charge was affirmed by the steady state anisotropy experiment. Fluorescence anisotropy provides a very good idea for the interaction between two (or more) species by looking into the changes in the motional restrictions. Limits to the motion of the fluorophore affects its fluorescence yield by modulating the radiative and non-radiative photophysical pathways. The changing trends in the steady state fluorescence anisotropy $(r$ ) of GSH-AuNCs with the DMTAP and DPPC GUVs (Fig. 3D) show that the binding of the AuNCs with the lipid headgroups is different in the two cases. The anisotropy increased gradually with the DMTAP GUVs, indicating progressively strong attachment. On the other hand, with DPPC GUVs, the anisotropy increased initially (in compliance with the fluorescence emission data) and subsequently saturated, thus substantiating the explanation stated above.

The results are further supported by the measured average zeta potential values on the addition of DMTAP and DPPC GUVS to the GSH-AuNCs (Fig. S2, ESI $\dagger$ and Table 1). Zeta potential

Table 1 Comparison of the zeta potentials of GSH-AuNCs on interaction with various concentrations of DMTAP and DPPC GUVs in aqueous buffer at $\mathrm{pH} 7.4$

\begin{tabular}{ll}
\hline Composition & Zeta potential $(\mathrm{mV})$ \\
\hline $20 \mu \mathrm{M}$ GSH-AuNC & -14.8 \\
With $5 \mu \mathrm{M}$ DMTAP & -18.6 \\
With $10 \mu \mathrm{M}$ DMTAP & -29.9 \\
With $5 \mu \mathrm{M}$ DPPC & -0.598 \\
With $10 \mu \mathrm{M}$ DPPC & 0.275
\end{tabular}


indicates the surface charge of the dispersed particles in a colloidal solution. We found that the zeta potential of the GSHAuNCs, which was originally $-14.8 \mathrm{mV}$, changed to $-29.9 \mathrm{mV}$ on addition of DMTAP GUVs, indicating higher accumulation of electrons towards the GSH-AuNCs due to the excitation of the system by the applied voltage, followed by transfer of electronic charge towards the AuNCs, thus making the surface of the conjugate more negative. On the other hand, on addition of the same concentration of DPPC GUVs, the value gradually became $0.275 \mathrm{mV}$, indicating induced electron deficiency since the electron cloud moves away from the surface of the DPPC GUV on excitation (also confirmed theoretically). Hence, the concentration of GSH changes with the electrostatic interaction between the AuNCs and GUVs. This reduces the surface negative charge of the DPPC GUV-GSH AuNC conjugate. Such an effect of the surface ligands on NCs was also reported earlier by Wu et al. ${ }^{29}$ To verify the situation, we did a control experiment by measuring the zeta potential of DMTAP and DPPC lipid vesicles without adding GSH-AuNCs (Fig. S3, ESI $\dagger$ ), where we saw the normal change in the zeta potential values according to the enhancement in the GUV population in solution. This made it clear that the change in the zeta potential values in the two cases is real and occurred due to the influence of the GUV-GSH AuNC conjugates.

Table 2 shows that the LMCT states of the GSH-AuNCs decay faster on interaction with the GUVs. ${ }^{28}$ The corresponding decay traces are provided in Fig. S4 (ESI $\dagger$ ). On changing the lipid from DMTAP to DPPC, the surface charge of the GSH-AuNCs interacting with the GUVs changes, resulting in a change in the energy of the LMCT states due to the differently charged lipid head groups. The lifetime decays indicate that the LMCT states of the GSH-AuNCs probably get more destabilized upon interacting with the DPPC GUVs, which stems from the existence of the nearby triplet states that allows intersystem crossing (ISC) (Scheme 2). Presumably, these triplet states were energetically unreachable before interacting with the GUVs. The values in

Table 2 The excited state lifetimes of GSH-AuNCs with and without the GUVs. The samples were excited at $375 \mathrm{~nm}$ and the $600 \mathrm{~nm}$ emission was monitored. Values in parentheses indicate the percent contribution to the fitting component and the $\chi^{2}$ values show the goodness of the fits. The data are within $\pm 5 \%$ error

\begin{tabular}{llllll}
\hline Composition & $\tau_{1}(\mathrm{ps})$ & $\tau_{2}(\mathrm{~ns})$ & $\tau_{3}(\mathrm{~ns})$ & $\tau_{\mathrm{av}}(\mathrm{ns})$ & $\chi^{2}$ \\
\hline GSH-AuNC & $490(3)$ & $7.0(10)$ & $119.4(87)$ & 118.6 & 1.11 \\
With 10 $\mu$ M DMTAP & $250(19)$ & $5.0(16)$ & $100.3(65)$ & 99.1 & 1.04 \\
With 10 $\mathrm{M}$ DPPC & $240(26)$ & $4.5(15)$ & $95.7(59)$ & 93.8 & 1.05
\end{tabular}

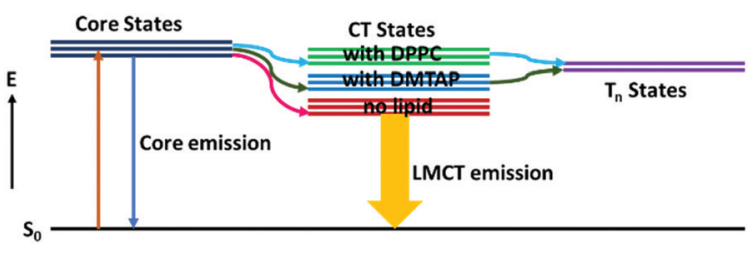

Scheme 2 Probable optical transitions in the GSH-AuNCs due to adsorption on the DMTAP and the DPPC GUVs.
Table 3 Various parameters for the GSH-AuNCs on interaction with the DMTAP and DPPC GUVs. The lipid concentration was $10 \mu \mathrm{M}$ in each case

\begin{tabular}{lllllll}
\hline Lipids & $\tau_{0}(\mathrm{~ns})$ & $\tau(\mathrm{ns})$ & $\bar{a}\left(\mathrm{~s}^{-1}\right)$ & $k_{\mathrm{nr}}\left(\mathrm{s}^{-1}\right)$ & $\Gamma\left(\mathrm{s}^{-1}\right)$ & $\Phi_{\text {eff }}(\%)$ \\
\hline DMTAP & 118.6 & 99.1 & $8.43 \times 10^{6}$ & $1.66 \times 10^{6}$ & $6.77 \times 10^{6}$ & 16.46 \\
DPPC & 118.6 & 93.8 & $8.43 \times 10^{6}$ & $2.23 \times 10^{6}$ & $6.20 \times 10^{6}$ & 20.93
\end{tabular}

parentheses in Table 2, which reflect the contributions of the respective components to the fit, indicate increase in LMCT on interaction with the DMTAP GUVs. More efficient ISC in case of the DPPC GUVs supports the inference. This is in concurrence with the previous reports ${ }^{28,29}$ and our experimental findings.

Numerical calculations provide the values of the respective responsible parameters. The rate of both the non-radiative and radiative energy transfers due to the interactions with the GUVs are calculated from the below eqn (1)-(4) and are summarized in Table $3 .^{30}$

$$
\begin{gathered}
k_{\mathrm{nr}}=\frac{1}{\tau}-\frac{1}{\tau_{0}} \\
\bar{a}=\tau_{0}{ }^{-1} \\
\bar{a}=\Gamma+k_{\mathrm{nr}} \\
\Phi_{\mathrm{eff}}=1-\frac{\tau}{\tau_{0}}
\end{gathered}
$$

Here, $\bar{a}$ is the decay rate of the fluorophore in absence of the GUVs, $\Gamma$ is the radiative rate, $k_{\mathrm{nr}}$ is the non-radiative decay rate of the fluorophore in presence of the GUVs, and $\Phi_{\text {eff }}$ is the efficiency of the non-radiative transfer of energy. $\tau_{0}$ and $\tau$ are the excited state lifetimes of the fluorophore in the absence and presence of the GUVs, respectively. The transfer rates and the $\Phi_{\text {eff }}$ values clearly indicate that ISC is higher on interaction of the GSH-AuNCs with the DPPC GUVs and hence clarify the effects of the differently-charged lipid head groups in the process.

\subsection{Modulation in the extent of photoinduced electron transfer in the presence of different GUVs}

Reports state that the surface ligands play a commanding role on the donor property of the NCs due to efficient LMCT. ${ }^{18,28,29}$ Also, the delocalized electrons donate directly to the metal core due to the influence of the nature of the surface attached ligands. ${ }^{29}$ Modulating the process of PET in the presence of suitable electron acceptors (or scavengers), such as $\mathrm{MV}^{2+}$, the GSH-AuNCs can become interesting candidates as light energy harvesters. ${ }^{28}$ Here, we applied $\mathrm{MV}^{2+}$ ions to initiate PET from the GSH-AuNCs attached to differently charged GUVs. The intention was to excavate the possibility of modulating the PET rate. Thiolated NCs reportedly reduce $\mathrm{MV}^{2+}$ to $\mathrm{MV}^{+} \bullet$ in the excited state. ${ }^{28,31,32}$ We explored the involvement of the GSH-AuNCs in PET with $\mathrm{MV}^{2+}$ in the absence and presence of DMTAP and DPPC GUVs at $\mathrm{pH}$ 7.4. Fluorescence from the GSH-AuNCs was differentially quenched by $\mathrm{MV}^{2+}$ in all the three cases (Fig. $4 \mathrm{~A}-\mathrm{C}$ ). Since $\mathrm{MV}^{2+}$ is a well-known electron acceptor and frequently used in PET experiments, hence, quenching 

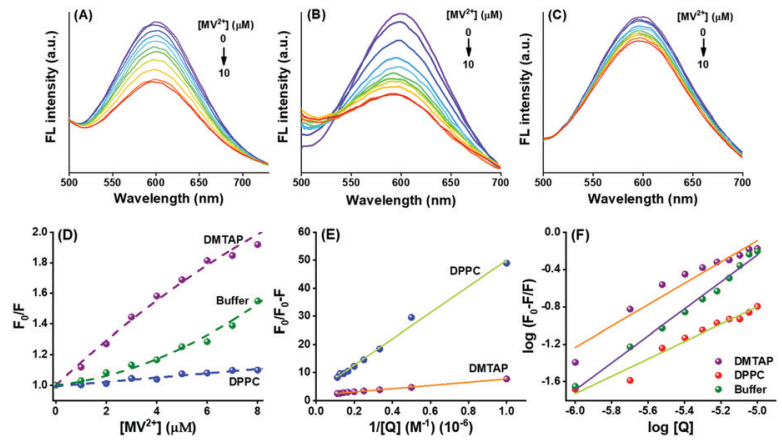

Fig. 4 Quenching of the fluorescence from GSH-AuNCs by the $\mathrm{MV}^{2+}$ ions in aqueous buffer ( $\mathrm{pH}$ 7.4) in (A) the absence of lipids, $(B)$ and $(C)$ the presence of the DMTAP and DPPC GUVs. The various plots with modified Stern-Volmer equations are given in (D) to (F) to obtain the different physical parameters arising due to the interactions as explained in the text

of fluorescence, in this case, can be well attributed to PET. The relative change in the fluorescence was fitted with non-linear regression in each case according to the modified Stern-Volmer equation as given by eqn (5):

$$
\frac{F_{0}}{F}=\left(1+K_{\mathrm{D}}[\mathrm{Q}]\right)\left(1+K_{\mathrm{S}}[\mathrm{Q}]\right)
$$

where, $F_{0}$ and $F$ are the fluorescence intensities in the absence and presence of the GUVs, $K_{\mathrm{D}}$ and $K_{\mathrm{S}}$ are the dynamic and static quenching constants, respectively, and [Q] is the concentration of the quencher, which suggests the existence of both dynamic and static quenching (Fig. 4D). The downward curvatures in the fits obtained with the GUVs indicate the inaccessibility of all the fluorophores to the quencher. This is inevitable since the $\mathbf{M V}^{2+}$ ions get distributed in the aqueous bulk as well as inside the GUV pool. ${ }^{33}$ Ions which are inside the aqueous pool do not reach the GSH-AuNCs to accept electrons through PET. The large difference in the extent of fluorescence quenching with DMTAP compared to the others indicates the extensive modulation of PET due to the GUVs. Following the explanations above, the plots in Fig. 4D show that PET is maximum with the DMTAP and minimum with the DPPC GUVs, respectively. Hence, the nature of the charged head groups of the lipids is considerably effective in the present case. Table 4 and Fig. S5 (ESI $\dagger$ ) provide the time-resolved decay parameters and the traces for the GSH-AuNCs on interaction with $\mathrm{MV}^{2+}$ in the absence and

Table 4 The excited state lifetimes of GSH-AuNCs with and without the GUVs in the presence and absence of $\mathrm{MV}^{2+}$. The samples were excited at $375 \mathrm{~nm}$ and the $600 \mathrm{~nm}$ emission was monitored. Concentration of GSHAuNCs in all the cases is $20 \mu \mathrm{M}$ and $6 \mu \mathrm{M}$ of $\mathrm{MV}^{2+}$ was added to them. Values in parentheses indicate the percent contribution to the fitting component and the $\chi^{2}$ values show the goodness of the fits. The data are within $\pm 5 \%$ error

\begin{tabular}{llllll}
\hline Composition & $\tau_{1}(\mathrm{ps})$ & $\tau_{2}(\mathrm{~ns})$ & $\tau_{3}(\mathrm{~ns})$ & $\tau_{\mathrm{av}}(\mathrm{ns})$ & $\chi^{2}$ \\
\hline GSH-AuNC & $490(3)$ & $7.0(10)$ & $119.4(87)$ & 118.6 & 1.11 \\
With MV $^{2+}$ & $460(5)$ & $6.1(13)$ & $112.2(82)$ & 111.19 & 1.09 \\
With DMTAP & $250(19)$ & $5.0(16)$ & $100.3(65)$ & 93.8 & 1.05 \\
With DMTAP + $\mathrm{MV}^{2+}$ & $280(19)$ & $5.0(21)$ & $88.3(60)$ & 86.5 & 1.12 \\
With DPPC & $240(26)$ & $4.5(15)$ & $95.7(59)$ & 93.8 & 1.05 \\
With DPPC + $\mathrm{MV}^{2+}$ & $250(25)$ & $4.9(19)$ & $94.5(56)$ & 92.87 & 1.05
\end{tabular}

Table 5 Various parameters as obtained from the quenching plots for the GSH-AuNCs with and without the GUVs in the presence of $6 \mu \mathrm{M} \mathrm{MV}^{2+}$

\begin{tabular}{llllll}
\hline Composition & $\tau_{\mathrm{o}} / \tau$ & $F_{\mathrm{o}} / F$ & $K_{\mathrm{D}}\left(\mathrm{M}^{-1}\right)$ & $K_{\mathrm{S}}\left(\mathrm{M}^{-1}\right)$ & $K_{\mathrm{q}}\left(\mathrm{M}^{-1} \mathrm{~s}^{-1}\right)$ \\
\hline GSH-AuNCs & 1.067 & 1.285 & $1.11 \times 10^{4}$ & $3.40 \times 10^{4}$ & $9.4 \times 10^{10}$ \\
With DMTAP & 1.146 & 1.815 & $2.43 \times 10^{4}$ & $9.73 \times 10^{4}$ & $24.5 \times 10^{10}$ \\
With DPPC & 1.010 & 1.080 & $0.16 \times 10^{4}$ & $1.16 \times 10^{4}$ & $1.78 \times 10^{10}$
\end{tabular}

presence of the DMTAP and DPPC GUVs. The $\tau_{\mathrm{av}}$ values clearly confirm the quenching of LMCT fluorescence of the GSH-AuNCs by $\mathrm{MV}^{2+}$. While adsorbed on the DMTAP GUVs, this effect is noticeably much pronounced compared to that of the DPPC GUVs.

The dynamic quenching constant $\left(K_{\mathrm{D}}\right)$, arising due to PET, is given by $K_{\mathrm{D}}=K_{\mathrm{q}} \tau_{0}$, where $K_{\mathrm{q}}$ is the bimolecular quenching constant and $\tau_{0}$ is the excited state lifetime of the GSH-AuNCs in absence of the quencher. $K_{\mathrm{q}}$ can be calculated from the collected lifetime data (Table 5) at a certain quencher concentration and hence, $K_{\mathrm{D}}$ can be found out in each case using the following eqn (6).

$$
\frac{\tau_{0}}{\tau}=1+K_{\mathrm{q}}[\mathrm{Q}]
$$

where, $\tau$ is the excited state lifetime of GSH-AuNCs in the presence of a fixed concentration of $\mathrm{MV}^{2+}$. Obtaining the values of $K_{\mathrm{q}}$ and $K_{\mathrm{D}}$ helps to find $K_{\mathrm{S}}$ using eqn (5). The calculated values are provided in Table 5 . Static quenching of energy occurs due to the electrostatic attachment of the $\mathrm{MV}^{2+}$ ions on the lipid heads and the GSH-AuNC surface, followed by dynamic PET. The order $\left(10^{10}\right)$ of $K_{\mathrm{q}}$ indicates diffusioncontrolled quenching in every case, assuring that binding between the fluorophore and the quencher facilitates fluorescence quenching. The largest value of $K_{\mathrm{q}}$ in DMTAP GUVs indicates stronger binding compared to the weaker DPPC GUV association due to the reasons explained earlier.

The Stern-Volmer equation is modified further to obtain the extent of accessibility of the $\mathrm{MV}^{2+}$ ions to the fluorophore under different conditions. The following eqn (7) was used for this purpose.

$$
\frac{F_{0}}{F_{0}-F}=\frac{1}{f_{\mathrm{a}} K_{\mathrm{a}}[\mathrm{Q}]}+\frac{1}{f_{\mathrm{a}}}
$$

where, $F_{0}$ and $F$ are the fluorescence intensities of the GSHAuNCs in the GUVs in the absence and presence of $\mathrm{MV}^{2+}$, respectively, $K_{\mathrm{a}}$ is the Stern-Volmer quenching constant of the accessible fraction and $f_{\mathrm{a}}$ is the fraction of the fluorophore accessible to the quencher. From Fig. $4 \mathrm{E}, f_{\mathrm{a}}$ was obtained from the intercepts and $K_{\mathrm{a}}$ from the slopes of the respective plots. The accessibility fractions $\left(f_{\mathrm{a}}\right)$ of the quencher in the presence of the DMTAP and the DPPC GUVs are 53.55\% and $93.49 \%$, respectively. The difference in the $f_{\mathrm{a}}$ values could be explained by the charges of the lipid head groups of DMTAP and DPPC. While DPPC has a phosphate group at the head, DMTAP contains an ammonium moiety. Hence, DPPC vesicles will attract more $\mathrm{MV}^{2+}$ ions and make them accessible to the AuNCs, and the DMTAP vesicles with positive surface charge will deter the access due to repulsion. The corresponding $K_{\mathrm{a}}$ values are $9.49 \times 10^{4} \mathrm{M}^{-1}$ and $2.44 \times 10^{4} \mathrm{M}^{-1}$. It is clear from 
these data that although accessibility of the $\mathrm{MV}^{2+}$ ions are much less than that to the DMTAP GUVs decorated with GSH-AuNCs, which are quenched due to PET, is much higher than that to the DPPC GUV-counterparts. This happens due to the surface ligand induced modulations to the GSH-AuNCs created by the different GUVs. This is in good agreement with the zeta potential results for the GSH-AuNCs adsorbed on DMTAP and DPPC (Table 1).

3.4. Modified Stern-Volmer equation to calculate the change in the Gibbs free energy for the electron transfer process

The Stern-Volmer equation is further modified to obtain eqn (8) to calculate the respective free energy changes, binding constants $\left(K_{\mathrm{b}}\right)$, and the number of binding sites $(n)$ for $\mathrm{GSH}$ AuNCs and the DMTAP and DPPC GUVs. ${ }^{34}$

$$
\log \left(\frac{F_{0}-F}{F}\right)=\log K_{\mathrm{b}}+n \log [\mathrm{Q}]
$$

The plot of $\log \left(F_{0}-F\right) / F$ vs. $\log [\mathrm{Q}]$ gives a straight line (Fig. $4 \mathrm{~F}$ ) with slope $\mathrm{n}$ and intercept $\log K_{\mathrm{b}}$.

The standard Gibbs free energy change for the process $\left(\Delta G^{0}\right)$ and that for the quenching process due to $\operatorname{PET}(\Delta G)$ are given by the equations: $\Delta G^{0}=-R T \ln K_{\mathrm{b}}$ and $\Delta G=\Delta G^{0}+-R T \ln [\mathrm{Q}]$. We took the $6 \mu \mathrm{M}$ quencher concentration for calculating the Gibbs free energies. The calculated parameters for the quenching processes in the absence and presence of the GUVs are given in Table 6. Negative $\Delta G$ values confirm the spontaneity of PET with or without GUVs in aqueous buffer. The values are more favorable for DMTAP compared to the DPPC GUVs, thus conforming with the discussions so far. The binding of $\mathrm{MV}^{2+}$ to the GSH-AuNCs is much stronger compared to that in the presence of the GUVs because of free diffusion and electrostatic attraction. However, the process in the presence of the DMTAP and DPPC GUVs is different due to the nature of the lipid head groups and favorable LMCT. Although the availability of the quenchers is less (53.55\%) to the DMTAP GUV-bound GSH-AuNCs due to favorable LMCT, we see better binding of the quenchers to the AuNC surface and hence better PET. The less favored PET with the AuNCs bound to the surface of the DPPC GUVs is evident from the lower binding constant value.

\subsection{Computational investigations to consolidate the observations}

We calculated the electron distribution in the highest occupied and the lowest unoccupied molecular orbitals (HOMO and LUMO) of the individual lipid molecules (DMTAP and DPPC)

Table 6 Binding constants, number of the binding sites, and changes in the Gibbs free energies due to PET between the GSH-NCs and the MV ${ }^{2+}$ ions in the presence and absence of the GUVs. The lipid concentration was $10 \mu \mathrm{M}$ in each case

\begin{tabular}{lllll}
\hline Compositions & $K_{\mathrm{b}}\left(\mathrm{M}^{-1}\right)$ & $n$ & $\Delta G^{0}\left(\mathrm{~kJ} \mathrm{Mol}^{-1}\right)$ & $\Delta G\left(\mathrm{~kJ} \mathrm{Mol}^{-1}\right)$ \\
\hline No lipid & $937.67 \times 10^{4}$ & 1.44 & -39.77 & -69.55 \\
DMTAP & $42.01 \times 10^{4}$ & 1.14 & -32.08 & -61.87 \\
DPPC & $0.697 \times 10^{4}$ & 0.92 & -21.92 & -51.71
\end{tabular}

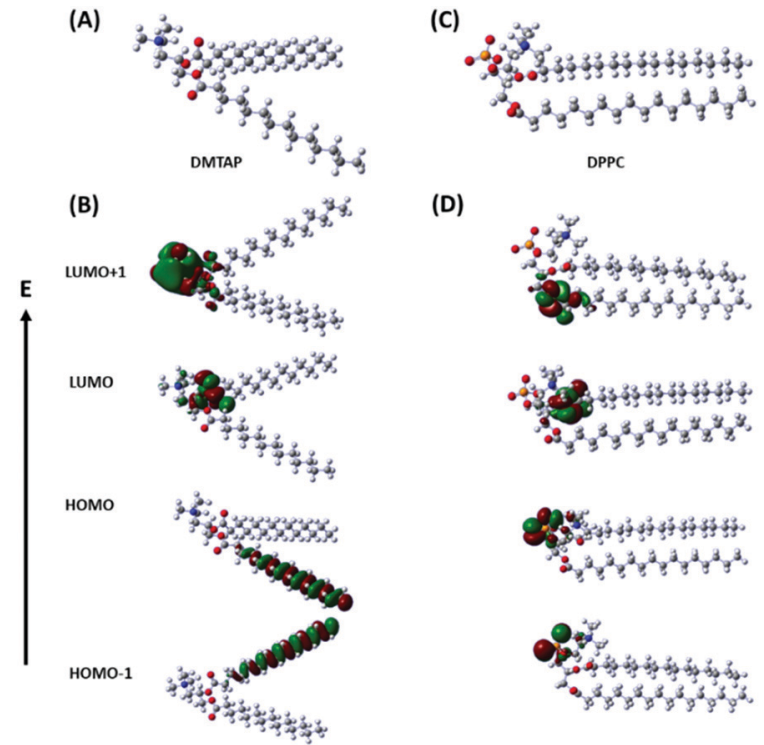

Fig. 5 Optimized geometries ( $A$ and $C$ ) and electron distributions in the HOMO, HOMO-1, LUMO, and LUMO+1 (B and D) for DMTAP and DPPC, respectively.

by solving their structures computationally using density functional theory (DFT) in the ground and excited states (Fig. 5). We have considered the electron density in the HOMO, HOMO-1, LUMO, and LUMO+1 in each case. The calculations were done using the $\omega$ B97X-D DFT functional since this, along with the empirical dispersion corrections, comprises $100 \%$ long-range exact exchange, which is about $22 \%$ of the shortrange exact exchange, an improved B97 exchange density functional for short-range interaction and the B97 correlation density functional. ${ }^{35}$ The basis function, 6-31G, has been used in the present calculations since this is generally used for atoms up to $\mathrm{Ar}^{36}$

The results show that the electron density mainly hovers over the two hydrophobic chains in HOMO and HOMO-1 for DMTAP, whereas, it shifts to the quaternary ammonium and the ester groups in LUMO and LUMO+1 on excitation by the incident radiation (375 nm) (Fig. 5B). For DPPC, the picture is very different due to the phosphate group adjacent to the quaternary amine part. The HOMO and HOMO-1 show that the electron density exists over the phosphate group that shifts downwards to the ester group in the LUMO and the LUMO+1 due to excitation (Fig. 5D). The results are extremely interesting since they corroborate very well with the descriptions that we proposed for the two types of GUVs influencing PET between the GSH-AuNCs and $\mathrm{MV}^{2+}$. The higher electron density towards the head region of the DMTAP GUVs induces greater LMCT to the AuNCs and hence converts them to better electron donors compared to the DPPC GUVs, where the effect is much lower.

\section{Conclusions}

We have synthesized GSH-coated AuNCs having negative surface charge, which interacted with DMTAP and DPPC lipidbased GUVs containing cationic and zwitterionic head groups, 
respectively. While DMTAP contains a quaternary ammonium group at the head, DPPC consists of a phosphate group after the quaternary ammonium moiety, followed by two hydrophobic tails. GSH-AuNCs are known to undergo PET with efficient electron scavengers, such as $\mathrm{MV}^{2+} ;^{25,28,32}$ however, modulation of the effect due to the adsorption of the GSHAuNCs over the lipid GUVs with different head charges has so far not been explored. This is important because GUVs are excellent mimics to biological cells. We observed from experiments as well as theoretical calculations that the electron density in DMTAP shifts towards the quaternary ammonium and the ester group in the excited state and in DPPC, the shift is away from the ammonium towards the ester group. This reinforces the GSH-AuNCs adsorbed over the DMTAP GUVs as better electron donors to the interacting $\mathrm{MV}^{2+}$ ions and hence follows much efficient PET compared to the DPPC GUVs. The dynamics were analyzed by fluorescence quenching due to PET. It is found that the attachment of the GSH-AuNCs with lipid vesicles possessing different head groups is very important in deciding the extent and occurrence of PET in aqueous buffer.

\section{Author contributions}

Arunavo Chatterjee - Conceptualization, data curation, formal analysis, investigation, methodology, software, validation, visualization, writing - original draft. Pradipta Purkayastha Conceptualization, formal analysis, funding acquisition, methodology, project administration, resources, supervision, validation, visualization, writing - review and editing.

\section{Conflicts of interest}

There are no conflicts to declare.

\section{Acknowledgements}

Financial support from the Science and Engineering Research Board (SERB), Department of Science and Technology, Government of India through the project CRG/2018/000555 is gratefully acknowledged. AC thanks SERB for his fellowship.

\section{Notes and references}

1 Y. Zhou, D. E. Harrison, K. Love-Myers, Y. Chen, A. Grider, K. Wickwire, J. R. Burgess, M. A. Stochelski and R. Pazdro, Biol. Med. C, 2014, 71, 157-164.

2 Z. Zhang, J. Jia, Y. Lai, Y. Ma, J. Weng and L. Sun, Bioorg. Med. Chem., 2010, 18, 5528-5534.

3 B. Harper, F. Sinche, R. H. Wu, M. Gowrishankar, G. Marquart, M. Mackiewicz and S. L. Harper, Nanomaterials, 2014, 4, 355-371.

4 C. A. Simpson, K. J. Salleng, D. E. Cliffel and D. L. Feldheim, Nanomedicine, 2013, 9, 257-263.

5 J. Liu, M. Yu, C. Zhou, S. Yang, X. Ning and J. Zheng, J. Am. Chem. Soc., 2013, 135, 4978-4981.
6 X.-D. Zhang, Z. Luo, J. Chen, S. Song, X. Yuan, X. Shen, H. Wang, Y. Sun, K. Gao, L. Zhang, S. Fan, D. T. Leong, M. Guo and J. Xie, Sci. Rep., 2015, 5.

7 G. Y. Tonga, K. Saha and V. M. Rotello, Adv. Mater., 2014, 26, 359-370.

8 K. P. García, K. Zarschler, L. Barbaro, J. A. Barreto, W. O'Malley, L. Spiccia, H. Stephan and B. Graham, Small, 2014, 10, 2516-2529.

9 K. Susumu, E. Oh, J. B. Delehanty, J. B. Blanco-Canosa, B. J. Johnson, V. Jain, W. J. Hervey, W. R. Algar, K. Boeneman, P. E. Dawson and I. L. Medintz, J. Am. Chem. Soc., 2011, 133, 9480-9496.

10 I.-I. S. Lim, D. Mott, W. Ip, P. N. Njoki, Y. Pan, S. Zhou and C.-J. Zhong, Langmuir, 2008, 24, 8857-8863.

11 M. Stobiecka, K. Coopersmith and M. Hepel, J. Colloid Interface Sci., 2010, 350, 168-177.

12 E. Moaseri, J. A. Bollinger, B. Changalvaie, L. Johnson, J. Schroer, K. P. Johnston and T. M. Truskett, Langmuir, 2017, 33, 12244-12253.

13 B. Weng, K.-Q. Lu, Z. Tang, H. M. Chen and Y.-J. Xu, Nat. Commun., 2018, 9, 1543.

14 Y. Negishi, M. Mizuno, M. Hirayama, M. Omatoi, T. Takayama, A. Iwase and A. Kudo, Nanoscale, 2013, 5, 7188-7192.

15 H. Chen, C. Liu, M. Wang, C. Zhang, N. Luo, Y. Wang, H. Abroshan, G. Li and F. Wang, ACS Catal., 2017, 7, 3632-3638.

16 B. Mondal, M. R. A. Raj and V. Ramamurthy, J. Chem. Sci., 2018, 130, 139.

17 Y.-S. Chen and P. V. Kamat, J. Am. Chem. Soc., 2014, 136, 6075-6082.

18 C. Talavera and P. V. Kamat, J. Chem. Sci., 2018, 130, 143.

19 G. Rossi and L. Monticelli, Biochim. Biophys. Acta, 2016, 1858, 2380-2389.

20 A. Ridolfi, L. Caselli, C. Montis, G. Mangiapia, D. Berti, M. Brucale and F. Valle, J. Microsc., 2020, 280, 194-203.

21 J. Sot, S. A. Mendanha-Neto, J. V. Busto, A. B. GarcíaArribas, S. Li, S. W. Burgess, W. A. Shaw, D. Gil-Carton, F. M. Goñi and A. Alonso, Chem. Phys. Lipids, 2019, 218, 40-46.

22 H. F. Salem, R. M. Kharshoum, H. A. Abou-Taleb and D. M. Naguib, J. Drug Targeting, 2019, 27, 1127-1134.

23 D. Bain, S. Maity and A. Patra, Phys. Chem. Chem. Phys., 2019, 21, 5863-5881.

24 M. Kizling, M. Dzwonek, A. Więckowska and R. Bilewicz, ChemCatChem, 2018, 10, 1988-1992.

25 K. G. Stamplecoskie and P. V. Kamat, J. Am. Chem. Soc., 2014, 136, 11093-11099.

26 A. M. Alkilany, S. Alsotari, M. Y. Alkawareek and S. R. Abulateefeh, Sci. Rep., 2019, 9, 11098.

27 S. Das and P. Purkayastha, Langmuir, 2017, 33, 7281-7287.

28 K. G. Stamplecoskie, Y.-S. Chen and P. V. Kamat, J. Phys. Chem. C, 2014, 118, 1370-1376.

29 Z. Wu and R. Jin, Nano Lett., 2010, 10, 2568-2573.

30 J. R. Lackowicz, Principles of Fluorescence Spectroscopy, Springer, New York, 3rd edn, 2006. 
31 S. Bhunia, S. Kumar and P. Purkayastha, ACS Omega, 2019, 4, 2523-2532.

32 S. Bhunia, S. K. Seth, P. Gupta, M. Karmakar, P. K. Datta and P. Purkayastha, ChemistrySelect, 2019, 4, 8568-8573.

33 S. Mondal, T. Das, A. Maity, S. K. Seth and P. Purkayastha, J. Phys. Chem. C, 2015, 119, 13887-13892.
34 R. Nasri, L. P. R. Bidel, N. Rugani, V. Perrier, F. Carrière, E. Dubreucq and C. Jay-Allemand, Molecules, 2019, 24, 2888.

35 J.-D. Chai and M. Head-Gordon, Phys. Chem. Chem. Phys., 2008, 10, 6615-6620.

36 V. A. Rassolov, J. A. Pople, M. A. Ratner and T. L. Windus, J. Chem. Phys., 1998, 109, 1223-1229. 